\title{
RADIOCARBON WIGGLE-MATCHING OF JAPANESE HISTORICAL MATERIALS WITH A POSSIBLE SYSTEMATIC AGE OFFSET
}

\author{
Mineo Imamura ${ }^{1,2} \bullet$ Hiromasa Ozaki $^{1} \bullet$ Takumi Mitsutani $^{3} \bullet$ Etsuko Niu $^{4} \bullet$ Shigeru Itoh $^{4}$
}

\begin{abstract}
Progress in radiocarbon accelerator mass spectrometry (AMS) techniques enables much more access to wiggle-matching techniques for high-precision ${ }^{14} \mathrm{C}$ dating with relatively low costs than before. Recently, we have applied wiggle-matching for a number of wood samples where dendrochronology is difficult because of various limitations imposed for dendro-dating. In most cases, wiggle-matching gave rather unambiguous calendar ages, but we found that in some cases the calibrated date was very sensitive to a systematic error of the ${ }^{14} \mathrm{C}$ date. Here, we present a wooden artifact from the Ujishigai archaeological site as a case where the highest wiggle-matched date did not agree with the date given by dendrochronology. An age with lower probability agreed with the tree-ring age of AD 389, which marked the beginning of the production of Sue ware (unglazed stoneware) in Japan. We show that systematic errors must be carefully taken into account while interpreting ${ }^{14} \mathrm{C}$ wiggle-matching results, whether they are due to instrumental errors (statistical) or due to a regional offset from the IntCal04 (Reimer et al. 2004) calibration curve.
\end{abstract}

\section{INTRODUCTION}

Wiggle-matching is increasingly important in the radiocarbon dating of historical materials, since recent accelerator mass spectrometry (AMS) techniques can provide high-precision measurements with relatively low costs. Wood is an ideal material for applying ${ }^{14} \mathrm{C}$ wiggle-matching, and there are numerous cases where dendrochronology cannot be applied because of rather stringent requirements needed for practical dating. Wood has been one of the most common materials used in dwellings, tools, crafts, etc., throughout our history, and wooden artifacts have often been found preserved in sites where swampy conditions prevented the sample from biological decay. ${ }^{14} \mathrm{C}$ wiggle-matching has been frequently applied to wooden artifacts and buildings in our research. In most cases, wigglematching gives a rather narrow range of age against the assumed additional errors. However, in some cases, calibrated dates are very sensitive to possible systematic errors in ${ }^{14} \mathrm{C}$ dates. In the following, we report the case of a wooden artifact excavated from the Uji-shigai archaeological site, Kyoto, Japan, where the second likely date with a much lower probability given by ${ }^{14} \mathrm{C}$ wigglematching (43 tree rings) coincides with the dendro-date (63 tree rings).

\section{MATERIALS AND ANALYSIS}

\section{Sample Description}

The wooden artifact used in this study was a processed board or an uncompleted tray, which was excavated in an undisturbed sediment layer of the site (Table 1), accompanied with the earliest type of Sue ware (unglazed stoneware) in Japan. Various artifacts were found undisturbed in the site, indicating that it had been occupied for a short time. The wood was Japanese cypress (Chamaecyparis obtusa or Hinoki cypress) and contained 63 tree rings, with the outermost tree ring accompanied by bark. These features reinforce the importance of the artifact as an absolute time marker for the beginning of Sue ware production in Japan.

\footnotetext{
${ }^{1}$ National Museum of Japanese History, The National Institute for Humanities, 117 Jonai-cho, Sakura-shi, Chiba 285-8502, Japan.

${ }^{2}$ Corresponding author. Email: imamura@ rekihaku.ac.jp.

${ }^{3}$ National Research Institute for Cultural Properties, Nara, 2-9-1 Nijo-cho, Nara-shi, Nara 630-8577, Japan.

${ }^{4}$ Paleo-Labo Co. Ltd., 1900-65 Shimotazawa, Kurohone-cho, Kiryu-shi, Gunma 376-0144, Japan.
} 
Table 1 Relevant data on the artifact studied in this work.

\begin{tabular}{ll}
\hline Sample & Processed wood \\
Name of site & Uji-shigai archaeological site \\
Location of site & 55 Myoraku, Uji, Kyoto, Japan \\
Structural remains & A-1 SD302, Middle layer \\
Date of collection & 10 October 2004 \\
\hline
\end{tabular}

Tree-ring dating was performed by comparing 63 ring patterns with a standard ring pattern available back to 912 BC for Japanese cypress developed by the National Research Institute for Cultural Properties, Nara (1990). Distinct ring patterns of Japanese cypress enabled dating with yearly precision.

In order to independently perform ${ }^{14} \mathrm{C}$ wiggle-match dating, a specimen including 43 tree rings was taken from an outside part of the wood, which included the outermost tree ring of the artifact. The sample specimen was sent to the National Museum of Japanese History, without any information on the dendro-date, and was divided into 5-yr blocks.

We also performed ${ }^{14} \mathrm{C}$ measurements for several reference wood samples around this period. Fiveyr blocks with known ages of AD 235 to 350 were taken from a dated wood from the Tohyamagawa riverbed (Nagano, Japan), $200 \mathrm{~km}$ east-northeast of Kyoto.

\section{Sample Pretreatment and AMS}

Several tens of milligrams of each tree-ring block were pulverized into submillimeter sizes and treated for conventional acid-alkali-acid (AAA) treatment by using an automatic AAA apparatus (Sakamoto et al. 2004). After drying overnight at $110^{\circ} \mathrm{C}$ in an electric oven, several milligrams of each treated sample were sealed into an evacuated quartz tube together with $\mathrm{CuO}$ (elementary analysis grade: Merck, Ltd.) and Sulfix ${ }^{\circledR}$ (elementary analysis grade: Wako Pure Chemical Industries, Ltd.). Sulfix (consisting of cobalt and silver oxides) was used for removing sulfur oxide and halogens. The samples were combusted for $3 \mathrm{hr}$ at $850{ }^{\circ} \mathrm{C}$ in sealed quartz tubes. The generated gases including $\mathrm{CO}_{2}$ were transferred into a vacuum line and then $\mathrm{CO}_{2}$ was purified cryogenically. The purified $\mathrm{CO}_{2}$ was converted to graphite at $600{ }^{\circ} \mathrm{C}$ by reducing with $\mathrm{H}_{2}$ in the presence of Fe powder (catalyst) to prepare the targets for AMS measurement.

The graphite targets were measured for ${ }^{14} \mathrm{C}$ with 6 NIST standards and 2 processed blanks using a compact AMS system at the Paleo-Labo Co. (Kobayashi et al. 2007). ${ }^{14} \mathrm{C}$ ages were normalized to the averaged data for 6 NIST standards. Reproducibility of the 6 standard samples was excellent, within the uncertainties given by the statistical errors of measurements $(0.25 \%)$. Correction for the contamination in preparation was made using processed blank samples. Blank correction was about $0.3 \%$ in the ${ }^{14} \mathrm{C} /{ }^{12} \mathrm{C}$ ratios for wood samples.

\section{Wiggle-Matching Analysis}

Wiggle-matching analysis was performed with Microsoft ${ }^{\circledR}$ Excel ${ }^{\circledR}$ software using Bayesian methods and the program file RHC3.2w (Imamura 2007), which has been developed for internal use to cope with various demands. The method of wiggle-matching is essentially the same as the one used in OxCal (Bronk Ramsey 1995, 2001; Bronk Ramsey et al. 2001), and the file includes several optional functions such as $\chi^{2}$ test analysis and mean value analysis.

In this work, probabilities were also calculated for a given offset from IntCal04 (Reimer et al. 2004). If ${ }^{14} \mathrm{C}$ ages for the $i$-th tree-ring are given $T_{i} \pm \sigma_{m i}$, and IntCal data given $f(t) \pm \sigma_{t}$, probability of the surface calendar age $t$ is approximated for an offset of $\alpha \pm \beta$ by the following equations: 


$$
P(t)=\Pi p\left(t-n_{i}\right) / \Sigma \Pi p\left(t-n_{i}\right)
$$

where $n_{i}=i$-th tree ring from the surface of wood; $p\left(t-n_{i}\right)$ is a probability at age $t$ imposed by the $i$-th tree ring, and

$$
\begin{gathered}
\left.p\left(t-n_{i}\right)=\left(2 \pi{\sigma^{\prime}}^{2}\right)^{1 / 2} \exp -\left(T_{i}-f^{\prime}\left(t-n_{i}\right)\right)^{2} / 2{\sigma^{\prime}}_{t}{ }^{2}\right) \\
f^{\prime}(t)=f(t)+\alpha \\
{\sigma^{\prime}}^{\prime}{ }^{2}=\sigma_{m i}{ }^{2}+\sigma_{t}{ }^{2}+\beta^{2}
\end{gathered}
$$

\section{RESULTS}

The results for the wooden artifact (WS) and reference tree rings (WR) are shown in Tables 2 and 3, respectively. It is confirmed that reference tree rings in this period give ${ }^{14} \mathrm{C}$ ages consistent with IntCal04 (Reimer et al. 2004) within the errors quoted.

Table 2 AMS ${ }^{14} \mathrm{C}$ results for the tree-ring blocks of the artifact. Tree rings are numbered 1 to 43 beginning with the outside ring.

\begin{tabular}{lclll}
\hline Sample name & $\begin{array}{l}\text { Tree-ring } \\
\text { number }\end{array}$ & $\begin{array}{l}{ }^{14} \mathrm{C} \text { age } \\
(\mathrm{BP} \pm 1 \sigma)\end{array}$ & $\delta^{13} \mathrm{C}^{\mathrm{a}}(\% \circ)$ & Lab code $^{\mathrm{b}}$ \\
\hline SGUS-1 & $1-5$ & $1741 \pm 21$ & $-26.35 \pm 0.14$ & PLD-4396 \\
SGUS-2 & $6-10$ & $1732 \pm 21$ & $-26.19 \pm 0.15$ & PLD-4397 \\
SGUS-3 & $11-15$ & $1740 \pm 21$ & $-25.56 \pm 0.14$ & PLD-4398 \\
SGUS-4 & $16-20$ & $1709 \pm 21$ & $-25.89 \pm 0.14$ & PLD-4399 \\
SGUS-5 & $21-25$ & $1703 \pm 21$ & $-25.77 \pm 0.16$ & PLD-4400 \\
SGUS-6 & $26-30$ & $1736 \pm 21$ & $-27.68 \pm 0.15$ & PLD-4401 \\
SGUS-7 & $31-35$ & $1671 \pm 21$ & $-27.62 \pm 0.14$ & PLD-4402 \\
SGUS-8 & $36-40$ & $1707 \pm 21$ & $-26.22 \pm 0.14$ & PLD-4403 \\
SGUS-9 & $41-43$ & $1712 \pm 22$ & $-27.03 \pm 0.13$ & PLD-4404 \\
\hline
\end{tabular}

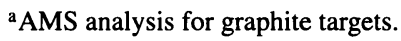

${ }^{b}$ Laboratory code of Paleo-Labo Inc.

Table 3 AMS ${ }^{14} \mathrm{C}$ results for reference samples.

\begin{tabular}{lllll}
\hline $\begin{array}{l}\text { Sample } \\
\text { name }\end{array}$ & Tree-ring age & $\begin{array}{l}{ }^{14} \mathrm{C} \text { age } \\
(\mathrm{BP} \pm 1 \sigma)\end{array}$ & $\delta^{13} \mathrm{C}(\%)$ & Lab code \\
\hline WR-8 & AD 231-235 & $1820 \pm 25$ & $-23.64 \pm 0.14$ & PLD-4191 \\
WR-1 & AD 251-255 & $1765 \pm 25$ & $-23.88 \pm 0.14$ & PLD-4017 \\
WR-2 & AD 266-270 & $1740 \pm 25$ & $-23.19 \pm 0.16$ & PLD-4018 \\
WR-3 & AD 281-285 & $1760 \pm 25$ & $-24.02 \pm 0.19$ & PLD-4019 \\
WR-4 & AD 296-300 & $1765 \pm 25$ & $-22.98 \pm 0.14$ & PLD-4020 \\
WR-5 & AD 316-320 & $1795 \pm 25$ & $-23.15 \pm 0.19$ & PLD-4021 \\
WR-6 & AD 321-325 & $1750 \pm 25$ & $-23.81 \pm 0.16$ & PLD-4022 \\
WR-9 & AD 341-345 & $1715 \pm 25$ & $-23.39 \pm 0.16$ & PLD-4192 \\
WR-7 & AD 346-350 & $1675 \pm 25$ & $-23.58 \pm 0.16$ & PLD-4023 \\
\hline
\end{tabular}


The ${ }^{14} \mathrm{C}$ results for 9 samples are matched to fit with the IntCal04 calibration curve. The results show 2 peaks at cal AD 295-317 (86\%) and cal AD 373-387 (9\%) at 95\% confidence limit, with the highest probability around cal AD 303. A $\chi^{2}$ test analysis gave similar results, however with wider ranges of estimated ages of cal AD 293-321 and cal AD 361-392.

As mentioned previously, dendrochronology was performed using an original artifact of 63 tree rings. The most probable tree-ring age was AD 389 by $t$ test $(t=4.9)$ for the outermost layer, in approximate agreement with the second peak around cal AD 383 in Figure 1, but not with the first peak. No other possibility existed except for $\mathrm{AD} 389$ within the range given by the ${ }^{14} \mathrm{C}$ dates.

Figure $1{ }^{14} \mathrm{C}$ wiggle-matching of the wooden artifact from Kyoto, Japan. The artifact provides a clue to determine the timing of the first Sue ware (unglazed stoneware) production in Japan. The results show 2 peaks at cal AD 295-317 (86\%) and cal AD 373-387 (9\%) at 95\% confidence limit, with the highest probability around cal AD 303 in conflict with the dendro-date of AD 389.

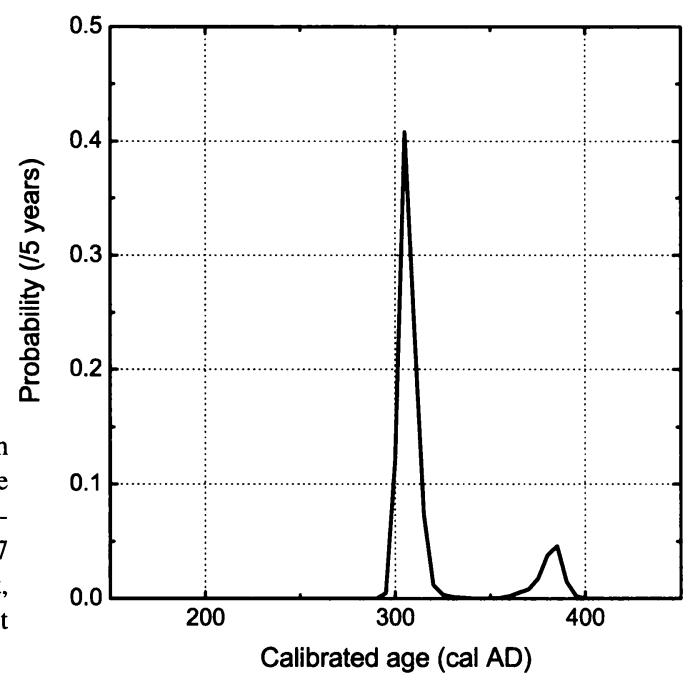

Assuming that the cal AD 303 peak is real, the sample data seem to be systematically younger by about $10-20{ }^{14} \mathrm{C}$ yr than $\mathrm{IntCal04}$ data and by $20-30{ }^{14} \mathrm{C}$ yr than those of the reference rings (Figure 2). This may mean that the cal AD 303 peak is not the true age for this sample. On the other hand, a small deviation is observed between the reference rings and IntCal04 as shown in the plots of Figure 2. The reference data consistently give slightly older ages than IntCal04.

The difference between the reference and IntCal04 data is calculated to be $14 \pm 7{ }^{14} \mathrm{C}$ yr based on the measured values of reference samples (Table 3) and the IntCal04 data, which is obtained by linear interpolation, with $1-\sigma$ standard errors. Taking the offset of $14 \pm 7{ }^{14} \mathrm{C} \mathrm{yr}$, we have performed wiggle-matching using Equations 1-4. The results are given in Figure 3 and show a single mode of distribution in the range of cal AD 372-398 (95\%), in excellent agreement with the dendro-age of AD 389 for the outermost tree ring.

The obtained age of the Uji-shigai archaeological site marks the time of the beginning of production of Sue ware (unglazed stoneware) in Japan, and is consistent with the dendro-dated age of AD 412, which was determined on a wooden artifact excavated with a slightly younger type of Sue ware (Mitsutani and Tsugiyama 1999). The age for the earliest type of Sue ware is about $50 \mathrm{yr}$ older than the traditional view.

\section{DISCUSSION}

As we see in Figures 2 and 3, the wiggle-matched sequence of ${ }^{14} \mathrm{C}$ dates from the wooden artifact in this work yields a bimodal age distribution, with the lower probability consistent with the dendro- 


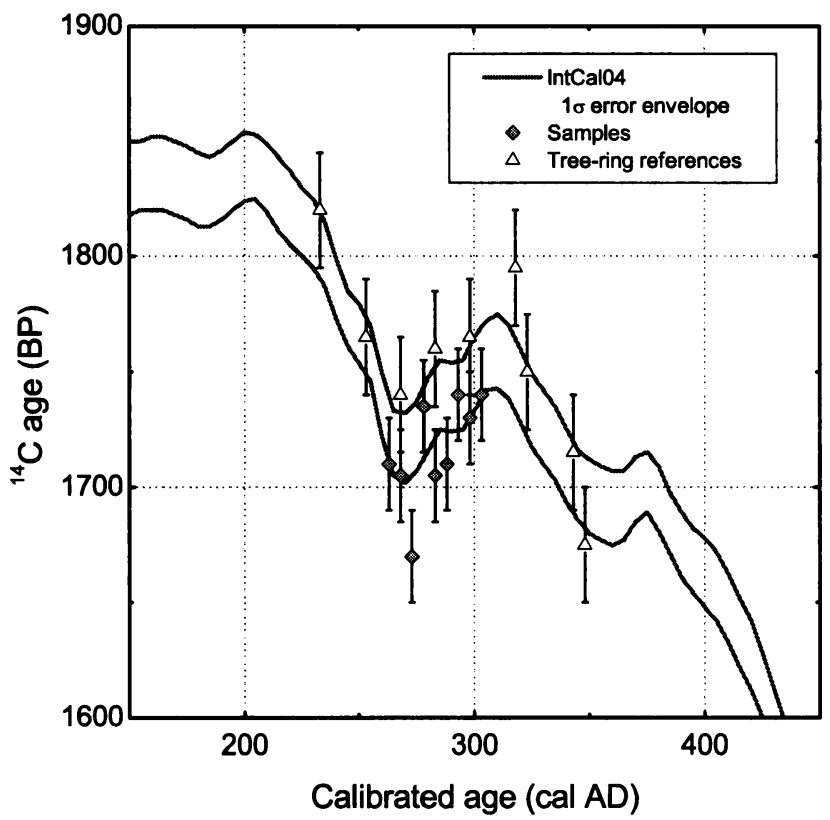

Figure 2 Measured ${ }^{14} \mathrm{C}$ data of the artifact are compared with IntCal04 and the ${ }^{14} \mathrm{C}$ ages of the references with known ages, assuming the outermost tree-ring age to be cal $\mathrm{AD} 303$.

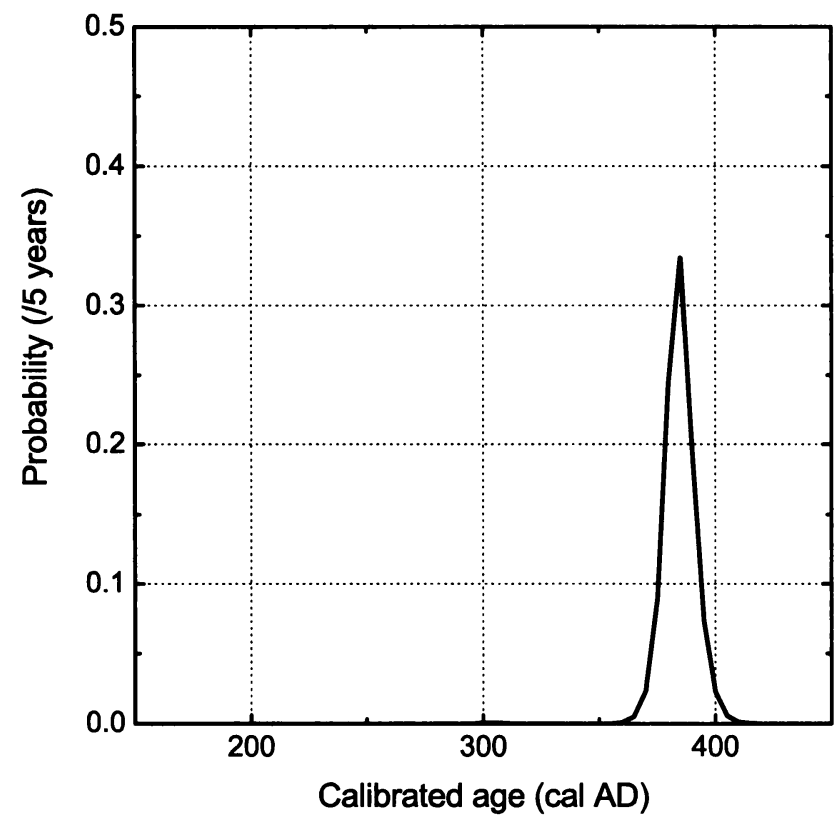

Figure 3 Probability distribution obtained for the artifact by wiggle-matching with a regional offset of $14 \pm 7{ }^{14} \mathrm{C} \mathrm{yr}$ applied. The age of cal AD 372$398(95 \%)$ is in an excellent agreement with the dendro-age of AD 389 for the outermost tree ring. 
date. However, by assuming a systematic error of $14 \pm 7{ }^{14} \mathrm{C}$ yr, which is estimated from the differences in ${ }^{14} \mathrm{C}$ age between IntCal04 and the reference rings with known ages, wiggle-matching gives a single-mode age distribution in excellent agreement with the dendro-date. This fact demonstrates the effectiveness of wiggle-matching and also shows that possible biasing or systematic error could occur in interpretation of ${ }^{14} \mathrm{C}$ wiggle-matching analysis.

The bimodal distribution of probability was mainly caused by the structure of wiggles around 1700 $1800{ }^{14} \mathrm{C}$ yr BP, and in part by the small number of tree rings (43 rings) used for ${ }^{14} \mathrm{C}$ analyses in this work. If a much longer section of wood sample were available, more accurate dates could have been obtained. However, it should be noted that a small systematic error of $10-20{ }^{14} \mathrm{C}$ yr could result in a wrong conclusion in some cases.

A source of the systematic error could be the small regional offset $\left(\sim 20{ }^{14} \mathrm{C} \mathrm{yr}\right)$ to an older age from IntCal04 in the above case. Regional offsets of $\sim 30{ }^{14} \mathrm{C} \mathrm{yr}$ from IntCal04 have been argued for Japanese wood samples in the range of AD 80-200 (Sakamoto et al. 2003), although overall ${ }^{14} \mathrm{C}$ data have shown consistent ages with IntCal04 between 820 BC and AD 900 (Sakamoto et al. 2003; Ozaki et al. 2007) (Figure 4).

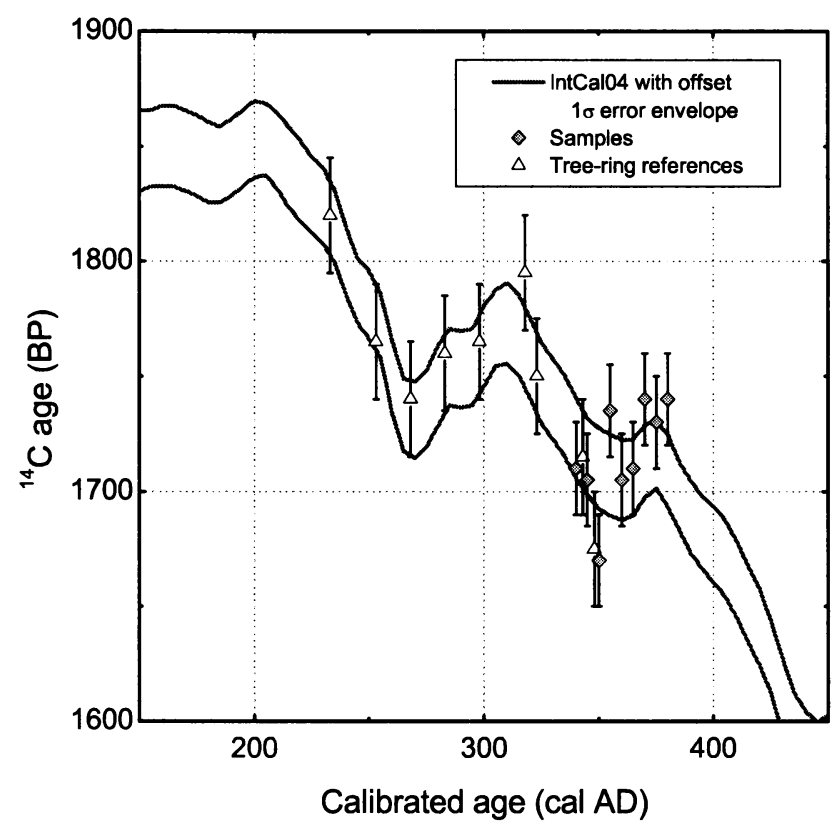

Figure 4 Measured ${ }^{14} \mathrm{C}$ data of the artifact are compared with IntCal04 corrected for a regional offset of $14 \pm 7{ }^{14} \mathrm{C} \mathrm{yr}$, and with the ${ }^{14} \mathrm{C}$ ages of the references with known ages, using the highest probability around cal $\mathrm{AD}$ 383 obtained in Figure 3.

Another possibility for the bimodal distribution is due to an experimental systematic error in the AMS measurements. The same effect as the regional offset is expected if the artifact sample data are shifted toward younger ages by $10-20{ }^{14} \mathrm{C}$ yr, as can be seen from Equations 2 and 3. This order of small deviation could occur even if several standards are used. In this work, $6{ }^{14} \mathrm{C}$ standard samples were measured with $0.25 \%(1 \sigma)$ counting statistics, so that the overall statistical fluctuation should be approximately $0.1 \%$, i.e. $\sim 10{ }^{14} \mathrm{C}$ yr $(1 \sigma)$. By taking $2-\sigma$ errors into account, a systematic deviation up to $20{ }^{14} \mathrm{C}$ yr might happen by chance in the normalization process of ${ }^{14} \mathrm{C}$ dates. 
As seen above, in some cases the calibrated date is very sensitive to a systematic error of the ${ }^{14} \mathrm{C}$ date. These errors must be carefully taken into account when interpreting results, whether they are instrumental (statistical) or due to a regional offset from the IntCal04 calibration data sets. In the above case, the regional offset is preferred to explain the observed deviations since it accounts for both cases of the artifact and the reference. Part of the problem may be solved by measuring, simultaneously with samples, ${ }^{14} \mathrm{C}$ in the reference tree rings with known ages of the same period, and by obtaining a systematic error from the difference between IntCal and the references. Correction for this systematic error should help improve the precision of ${ }^{14} \mathrm{C}$ wiggle-matching.

\section{ACKNOWLEDGMENTS}

We are indebted to the Board of Education, Uji-shi, Kyoto, for providing us with the wood sample used in this work and relevant archaeological information. We are particularly grateful to $C$ Bronk Ramsey for many useful comments and suggestions, and also to an anonymous reviewer for comments in improving the paper. Thanks are also due to T Shinmen and I Nanbu for technical assistance. This work was supported by a Grant-in-Aid for Creative Scientific Research of the Japan Society for the Promotion of Science (\#16GS0118).

\section{REFERENCES}

Bronk Ramsey C. 1995. Radiocarbon calibration and analysis of stratigraphy: the OxCal program. Radiocarbon 37(2):425-30.

Bronk Ramsey C. 2001. Development of the radiocarbon calibration program. Radiocarbon 43(2A):355-63.

Bronk Ramsey C, van der Plicht J, Weninger B. 2001. 'Wiggle matching' radiocarbon dates. Radiocarbon 43(2A):381-9.

Imamura M. 2007. On the radiocarbon calibration program file, RHC3.2. Bulletin of the National Museum of Japanese History 137. In Japanese.

Kobayashi K, Niu E, Itoh S, Yamagata H, Lotaidze Z, Jorjliani I, Nakamura K, Fujine H. 2007. The compact ${ }^{14} \mathrm{C}$ AMS facility of Paleo Labo Co., Ltd., Japan. $\mathrm{Nu}$ clear and Instrumental Methods in Physics Research B 259(1):31-5.

Mitsutani T, Tsugiyama J. 1999. Wooden artifacts from Kofun-period layers below the Nara palace site and its Dendrochronological dating. Annual Bulletin of Nara National Cultural Properties Research Institute 1999I. p 8-9.

National Research Institute for Cultural Properties, Nara. 1990. Dendrochronology in Japan. Research report
\#48 of the National Research Institute for Cultural Properties, Nara.

Ozaki H, Imamura M, Matsuzaki H, Mitsutani T. 2007. Radiocarbon in 9th to 5th century BC tree-ring samples from the Ouban 1 archaeological site, Hiroshima, Japan. Radiocarbon, these proceedings.

Reimer PJ, Baillie MGL, Bard E, Bayliss A, Beck JW, Bertrand CJH, Blackwell PG, Buck CE, Burr GS, Cutler KB, Damon PE, Edwards RL, Fairbanks RG, Friedrich M, Guilderson TP, Hogg AG, Hughen KA, Kromer B, McCormac G, Manning S, Bronk Ramsey C, Reimer RW, Remmele S, Southon JR, Stuiver M, Talamo S, Taylor FW, van der Plicht J, Weyhenmeyer CE. 2004. IntCal04 terrestrial radiocarbon age calibration, 0-26 cal kyr BP. Radiocarbon 46(3):1029-58.

Sakamoto M, Imamura M, van der Plicht J, Mitsutani T, Sahara M. 2003. Radiocarbon calibration for Japanese wood samples. Radiocarbon 45(1):81-9.

Sakamoto M, Kodaira A, Imamura M. 2004. An automated AAA preparation system for AMS radiocarbon dating. Nuclear and Instrumental Methods in Physics Research B 223-224:298-301. 